\title{
Bayesian inference for multistage and other incomplete designs
}

\author{
Jesse Koops, Timo Bechger, and Gunter Maris
}

Affiliation

\begin{abstract}
Following Maris, Bechger and San-Martin (2015), we develop a Markov chain - Monte Carlo method for Bayesian inference tailored to handle data collected in multistage and other incomplete designs. We illustrate its operating characteristics with simulated data, and provide a real application.
\end{abstract}

\section{Introduction}

Building on work by Maris, Bechger, and San-Martin (2015), we propose a new Data Augmentation (DA) Gibbs sampler (Tanner \& Wong, 1987) for a large class of Item Response Theory (IRT) models. We focus on the Nominal Response Model (NRM; Bock, 1972) when the scoring parameters for the response categories of an item are known integers. The algorithm is tailor-made for incomplete designs and particularly suited for the calibration of large-scale test administrations as its com- 
putational cost does not depend on the number of respondents, and the estimation of item-category thresholds is robust against misspecification of the ability distribution.

We first introduce the marginal and extended NRM, following Maris et al. (2015) and Cressie and Holland (1983). We then derive the DA-Gibbs sampler. Subsequently, we discuss the adaptations needed to deal with multistage testing data and provide a real data example to illustrate that our approach can support a largescale testing program. All computations are done using $\mathrm{R}$ packages dexter (Maris, Bechger, Koops, \& Partchev, 2019) and dexterMST (Bechger, Koops, Partchev, \& Maris, 2019). An appendix provides some background to make the chapter more self-contained.

\section{The marginal and the extended NRM}

Let $^{1} x_{p i j}$ denote the dummy-coded response to item $i$ by person $p$; that is, $x_{p i j}=1$ if person $p$ picked category $j$ of item $i$. Let $a_{i j}$ denote the number of points earned for a response in category $j$. These item-category scores are assumed to be integer-valued and known with the lowest score equal to 0. Suppressing the person index, the manifest probabilities for a marginal NRM are:

$$
p_{f}(\mathbf{x} \mid \mathbf{b}, \mathbf{a})=\int_{0}^{\infty} \prod_{i} \frac{\prod_{j}\left(b_{i j} t^{a_{i j}}\right)^{x_{i j}}}{1+\sum_{j} b_{i j} t^{a_{i j}}} f(t) d t
$$

where $t>0$ denotes student ability with density $f$, and $b_{i j}>0$ is the parameter of category $j$ of item $i$. Given that practitioners choose the category scores in advance,

\footnotetext{
${ }^{1}$ When possible without causing confusion we will not distinguish explicitly between random variables and their realization.
} 
the model is a slight extension of the one-parameter logistic model (OPLM) (Verhelst \& Glas, 1995) and encompasses the Rasch model and the partial credit model ${ }^{2}$.

Following the derivation (see Appendix) of the extended Rasch model in Maris et al. (2015), we can re-write the manifest probabilities in (1) as:

$$
p(\mathbf{x} \mid \mathbf{b}, \boldsymbol{\lambda}, \mathbf{a})=\frac{\left(\prod_{i} \prod_{j} b_{i j}^{x_{i j}}\right) \lambda_{x_{++}}}{\sum_{s} \gamma_{s}(\mathbf{b}) \lambda_{s}}
$$

where $x_{++}=\sum_{i} \sum_{j} a_{i j} x_{i j}$ is the test score. These correspond to a marginal NRM if, and only if, the $\lambda_{s}$ are moments of the ability distribution. We will not require this and therefore refer to the manifest probabilities in Equation 2 as the extended NRM (eNRM)

The functions $\gamma_{s}$ play an important role in what follows. They are defined as:

$$
\gamma_{x_{++}}(\mathbf{b})=\sum_{\mathbf{x} \rightarrow x_{++}} \prod_{i} \prod_{j} b_{i j}^{x_{i j}}
$$

where $\mathbf{x} \rightarrow x_{++}$means summation over all response vectors $\mathbf{x}$ leading to a test score $x_{++}$, and $\gamma_{s}$ is defined to be zero whenever $s$ is not a possible value of $x_{++}$. Note that the $\gamma$-functions are a direct extension of the elementary symmetric functions (e.g., Verhelst, Glas, \& van der Sluis, 1984), and they share with these a simple recursive property. That is, for any item $i$ :

$$
\gamma_{s}(\mathbf{b})=\gamma_{s}\left(\mathbf{b}_{(i)}\right)+\sum_{j} \gamma_{s-a_{i j}}\left(\mathbf{b}_{(i)}\right) b_{i j}
$$

\footnotetext{
${ }^{2}$ This may be more easily seen in the more popular parameterization with ability $\theta=\log (t)$ and item-category parameters $\eta_{i j}=-\log \left(b_{i j}\right)$.
} 
where $\mathbf{b}_{(i)}$ denotes the item parameters without the parameters belonging to item $i$. The recursion provides an efficient method to compute the $\gamma$ functions, and shows that each $\gamma$ function is linear in each of the item-category parameters $b_{i j}$.

An incomplete design presents different subsets of items, called test forms or "booklets", to different test takers giving incomplete data. To deal with such data, we consider the manifest probabilities given a design matrix $\mathbf{D}$, with elements $d_{p i}$ equal to one whenever person $p$ responded to item $i$, and zero otherwise. For a sample of students, the likelihood is:

$$
p(\mathbf{x} \mid \mathbf{b}, \boldsymbol{\lambda}, \mathbf{a}, \mathbf{D})=\frac{\prod_{i} \prod_{j} b_{i j}^{x_{+i j}} \prod_{k} \prod_{s} \lambda_{k s}^{n_{k s}}}{\prod_{k}\left(\sum_{s} \gamma_{s}\left(\mathbf{b}^{(k)}\right) \lambda_{k s}\right)^{n_{k+}}}
$$

where $x_{+i j}=\sum_{p} a_{i j} x_{p i j} d_{p i}, n_{k s}$ denotes the number of students with score $s$ on booklet $k$, and $n_{k+}=\sum_{s} n_{k s}$ is the total number of students that responded to test form $k$. Note that $s$ now runs over scores on test-form $k$.

\section{Bayesian inference for the eNRM}

We adopt a gamma prior $^{3}$ for every parameter: $\Lambda_{k s} \sim \operatorname{Gamma}\left(\nu_{k s}, \sigma_{k s}\right)$ and $B_{i j} \sim \operatorname{Gamma}\left(\eta_{i j}, \rho_{i j}\right)$, to obtain the following posterior distribution:

$$
f(\mathbf{b}, \boldsymbol{\lambda} \mid \mathbf{x}, \mathbf{a}, \mathbf{D}) \propto \frac{\prod_{i} \prod_{j} b_{i j}^{x_{i j}+\eta_{i j}-1} e^{-\rho_{i j} b_{i j}} \prod_{k} \prod_{s} \lambda_{k s}^{n_{k s}+\nu_{k s}-1} e^{-\sigma_{k s} \lambda_{k s}}}{\prod_{k}\left(\sum_{s} \gamma_{s}\left(\mathbf{b}^{(k)}\right) \lambda_{k s}\right)^{n_{k+}}} .
$$

We propose a DA Gibbs sampler to generate a Markov chain for sampling from this posterior. The key ingredient is the well-known (gamma) integral equation:

$$
\frac{\Gamma(n)}{\lambda^{n}}=\int_{\mathbb{R}^{+}} y^{n-1} \exp (-\lambda y) d y
$$

\footnotetext{
${ }^{3} \operatorname{Gamma}(\alpha, \beta)$ denotes a gamma distribution with shape $\alpha>0$ and rate $\beta>0$.
} 
where $\Gamma(n)=(n-1)$ ! denotes the gamma function. Using this integral equation for every factor in the denominator of the posterior distribution (5) gives the following:

$$
\begin{aligned}
f(\mathbf{b}, \boldsymbol{\lambda} \mid \mathbf{x}, \mathbf{a}, \mathbf{D}) & \propto \int_{\mathbb{R}_{+}^{K}} \prod_{i} \prod_{j} b_{i j}^{x_{+i j}+\eta_{i j}-1} \exp \left(-\rho_{i j} b_{i j}\right) \\
& \times \prod_{k} \prod_{s} \lambda_{k s}^{n_{k s}+\nu_{k s}-1} \exp \left(-\sigma_{k s} \lambda_{k s}\right) \\
& \times \prod_{k} y_{k}^{n_{k+}-1} \exp \left(-\left(\sum_{s} \gamma_{s}\left(\mathbf{b}^{(k)}\right) \lambda_{k s}\right) y_{k}\right) d \mathbf{y}
\end{aligned}
$$

where $K$ denotes the number of test forms. The expression inside the integral is (proportional to) the augmented posterior distribution $f(\mathbf{b}, \boldsymbol{\lambda}, \mathbf{y} \mid \mathbf{x}, \mathbf{a}, \mathbf{D})$ with latent data $\mathbf{y}$. Since the $\gamma$ function is linear in each of its arguments, it is straightforward to sample from this distribution using a Gibbs sampler. Specifically, all full conditional distributions are gamma:

$$
\begin{aligned}
Y_{k} \mid \ldots & \sim \operatorname{Gamma}\left(n_{k+}, \sum_{s} \gamma_{s}\left(\mathbf{b}^{(k)}\right) \lambda_{k s}\right) \\
\Lambda_{k s} \mid \ldots & \sim \operatorname{Gamma}\left(n_{k s}+\nu_{k s}, \gamma_{s}\left(\mathbf{b}^{(k)}\right) y_{k}+\sigma_{k s}\right) \\
B_{i j} \mid \ldots & \sim \operatorname{Gamma}\left(x_{+i j}+\eta_{i j}, \sum_{k} y_{k} \sum_{s \geq a_{i j}} \gamma_{s-a_{i j}}\left(\mathbf{b}_{(i)}^{(k)}\right) \lambda_{k s}+\rho_{i j}\right)
\end{aligned}
$$

where we use the shorthand notation ... to refer to all other random variables in the joint distribution. The resulting DA-Gibbs sampler is implemented in the dexter package.

\section{Operating characteristics}

DA increases auto-correlation. However, with only one latent variable per test form, the increase should not be large, compared to a Gibbs sampler for the posterior 

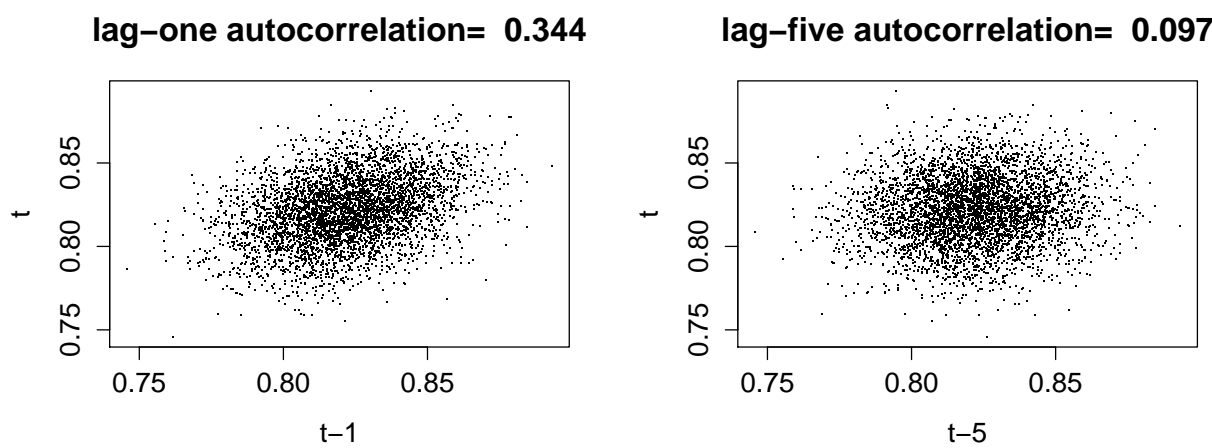

Figure 1. Lag one (left panel) and five (right panel) auto-correlation for a simulated example with 80 3-category items in 3 test forms of 40 items each, and a sample size of 20000 persons per test form

distribution itself. To illustrate, Figure 1 shows the auto-correlation for a simulated data set of 80 items, with three categories each (scored as $0,1,3$ ), in three test forms of 40 items each and a sample size of 20000 persons per test form.

We see that in comparison to the results on auto-correlation for a complete data eRM presented by Maris et al. (2015), the auto-correlation is indeed larger. However, looking at the lag five auto-correlation, we see that the auto-correlation at lag five is close to zero. Other operating characteristics are similar to those reported by Maris et al. (2015) with convergence being achieved after only a hundred iterations.

\section{Multi-Stage Testing}

Multi-Stage Testing (MST) is an early attempt to achieve adaptivity in testing (Bejar, 2014). In MST, items are selected for administration not separately but in (non-overlapping) bunches, usually called modules. In the first stage, all respondents 


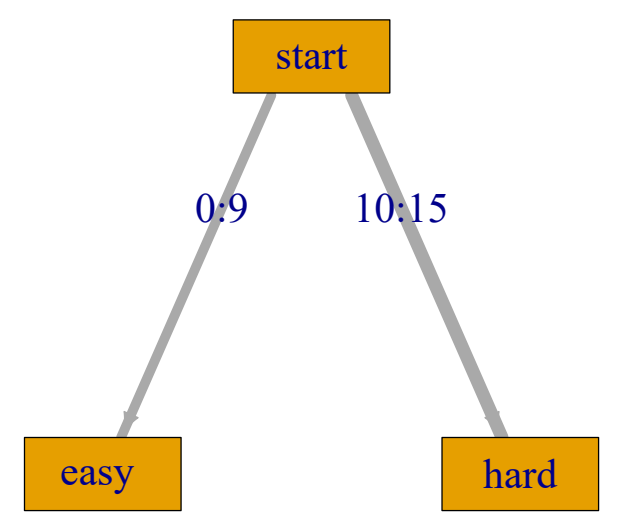

Figure 2. A two-stage MST design. The root represents the first stage where all examinees take a routing test. In the second stage, examinees with a score lower than or equal to 9 on the routing test take the easy module. Others take the hard module. The thickness of a path indicates how many respondents took it.

take a routing test. In subsequent stages, the modules they are given depend on their success in previous modules: test takers with high scores are given more difficult modules, and those with low scores are given easier ones; see e.g., Zenisky, Hambleton, and Luecht (2009), Hendrickson (2007), or Yan, Lewis, and von Davier (2014). To illustrate, Figure 2 shows a diagram of the simplest two-stage design. Here, the booklet consisting of modules "start" and "easy" is the easier booklet.

The DA Gibbs sampler, as it stands, will give biased results when applied to MST data unless we condition on the design (Zwitser \& Maris, 2015). More specifically, the likelihood must be adapted to take into account that certain responses patterns are excluded by the restrictions on (module) scores that are imposed by the 
design. If, for example, a persons took the easy booklet, responses with ten or more correct answers on the routing test are impossible. This must be discounted in the normalizing constant of the manifest probabilities and results in minor changes in the calculation of the $\gamma$-functions; the same as needed for CML estimation. The Appendix explains this point assuming a Rasch model.

Given that the items are partitioned between modules, we obtain the $\gamma$-function for a booklet, $\gamma_{s}\left(\mathbf{b}^{(k)}\right)$, by "merging" the $\gamma$-functions calculated for each module in the booklet; taking into account that some scores are ruled-out by the routing rules. For the easy booklet in Figure 2, for example, this merge-operation ${ }^{4}$ would be:

$$
\gamma_{s}\left(\mathbf{b}^{(1)}\right)=\sum_{s_{1}+s_{2}=s} \gamma_{s_{1}}\left(\mathbf{b}^{[\text {start }]}\right) \gamma_{s_{2}}\left(\mathbf{b}^{[\text {easy }]}\right), \quad s_{1} \in \mathcal{R}_{\text {start }}^{(1)}, s_{2} \in \mathcal{R}_{\text {easy }}^{(1)}
$$

where $\mathbf{b}^{[m]}$ are the parameter of the items in module $m, s_{m}$ is the score obtained on module $m$, and $\mathcal{R}_{m}^{(1)}$ the set of possible value of $s_{m}$ in booklet 1 . In this case, $\mathcal{R}_{\text {start }}^{(1)}$ contains scores 0 to 9 while scores on the last module are unrestricted. When there are more than two modules, merging can be done recursively; that is, we first merge the first and second module, then merge this with the third module, etc. A general implementation can be found in the dexterMST package.

Note that a linear test can be considered a MST with unrestricted module scores. Thus, the DA Gibbs sampler for MST can handle designs that include combinations of linear and multistage tests.

\footnotetext{
${ }^{4}$ Note that the recursion (3) is a special case where one module consists of a single item.
} 


\section{Real data application}

To illustrate that the DA Gibbs sampler can support a large testing program, we calibrate data from one form of the $\mathrm{ACT}^{5}$ (ACT, 2019) assessment English. The English Reading test consists of 75 items and was taken by 337,318 students. The ACT is administered as a linear test and for our present purpose we created a MST design by removing subsets of responses from the complete data. A complication was that the items are divided in five testlets, each consisting of a reading passage and 15 items. These testlets cannot be split and we opted for the simple two-stage design shown in Figure 2. A random sample of 318 persons was used to mimic a pretest which provided a rough estimate of item difficulty that was used to compose the modules: a medium (start) module consisting of one passage and two follow up modules, easy and hard, consisting of two passages each.

The DA Gibbs sampler did the job fast enough. After loading the data in memory, 1000 iterations took approximately one second on a 2.9 Ghz 4 core Windows laptop, compared to 0.06 seconds for CML calibration and two minutes for MML calibration using the mirt package (Chalmers, 2012).

Model fit was evaluated at the level of passage scores. Specifically, we looked

\footnotetext{
${ }^{5}$ Originally an abbreviation of American College Testing Program.
} 

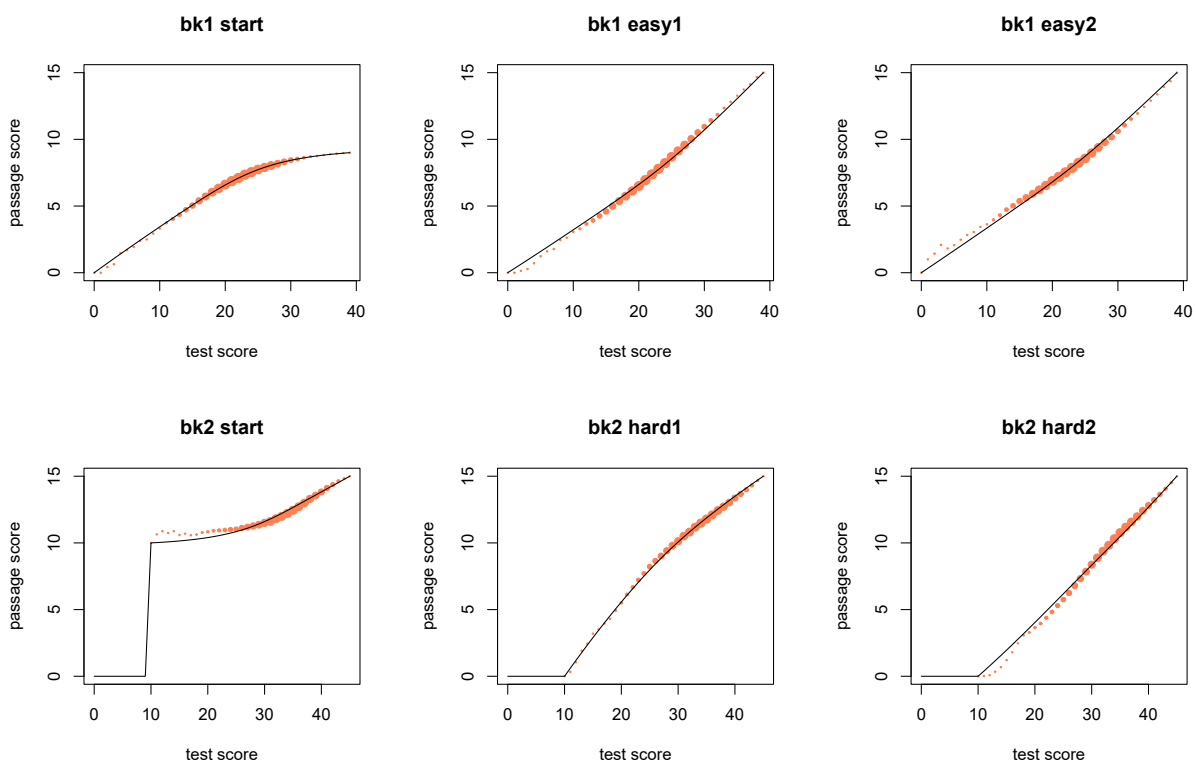

Figure 3. Passage-total score regressions based on MST calibration. The lines are the regressions under the model. The dots show the data; their size reflecting the number of observations.

at the distribution of passage scores, conditionally on the total score ${ }^{6}$ :

$$
\begin{aligned}
p\left(X_{++}^{[\text {start }]}=s_{1}, X_{++}^{[\text {easy 1] }}=s_{2}, X_{++}^{[\text {easy 2] }}=s_{3} \mid X_{++}=\right. & \left.s_{1}+s_{2}+s_{3}\right)= \\
& \frac{\gamma_{s_{1}}\left(\mathbf{b}^{[\text {start }]}\right) \gamma_{s_{2}}\left(\mathbf{b}^{[\text {easy 1] }}\right) \gamma_{s_{3}}\left(\mathbf{b}^{[\text {easy 2] }}\right)}{\gamma_{s_{1}+s_{2}+s_{3}}\left(\mathbf{b}^{(1)}\right)}
\end{aligned}
$$

which is defined only for admissible combinations of passage scores. This expression allows us to compute the passage-total-score regression functions implied by the model. Figure 3 shows that these match closely to the data. A calibration of the complete data gave similar results but misfit is visible when we zoom in on items that respondents did not take in the MST design. This is illustrated in Figure 4 which

\footnotetext{
${ }^{6}$ For our present purpose, we use the number correct score rather than the ACT scale score.
} 


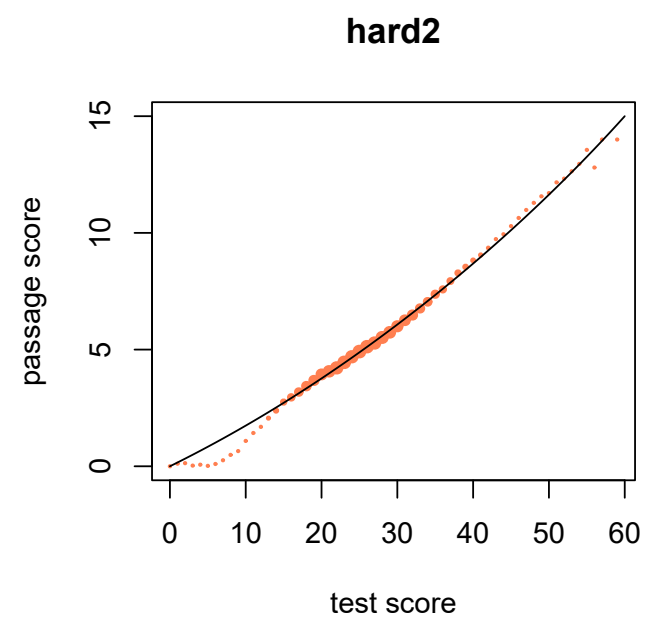

Figure 4. Passage-total-score regression for the hard module. CML on complete data of persons who took the easy booklet in the MST; excluding the routing module.

shows that, contrary to expectations, there is no evidence that less able respondent take refuge to guessing on hard items: instead, they seem to skip them, only gaining points on the easy items.

Practical differences between the linear and the, considerably shorter, multistage test are quite small. IRT-based reliability ${ }^{7}$ of the score on the full test was estimated to be 0.94 when we calibrated the complete data, compared to 0.95 when we used the MST data. We attribute this difference to the aforementioned model misfit in the complete data. Weighted ML estimates of ability correlated 0.97 ; with a reliability of 0.91 for the MST and 0.93 for the linear test (van Rijn, 2014). Standard errors are

\footnotetext{
${ }^{7}$ This is calculated as the correlation between scores generated under the model; see e.g., Bechger, Maris, Verstralen, and Béguin (2003).
} 


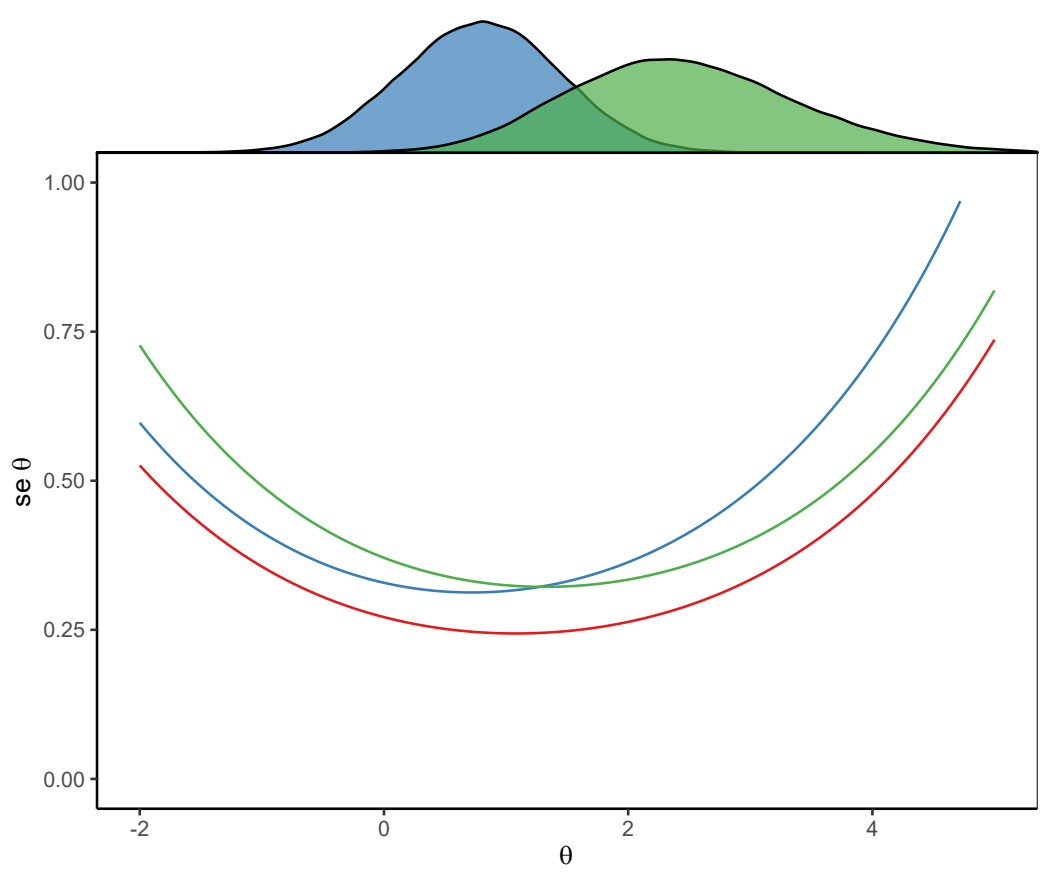

Figure 5. Standard errors of MLE estimates of ability for the easy (blue) and hard (green) MST booklets and the complete test (red). Estimated ability distributions for those who took the easy booklet (blue) and the hard booklet (green) are drawn in the margin.

shown in Figure 5 where we can see that estimation is only slightly more precise with the longer linear test.

It is worth noting that the ACT test not only serves for college admission but can also be used in state models for federal and state accountability. In this context, it is not the individual ability estimates that are of interest, but rather the distribution of ability for relevant sub-populations. For survey applications like this, plausible values are the method of choice, both from a practical and a theoretical point of view 
(Marsman, Maris, Bechger, \& Glas, 2016). Figure 6 shows the ability distribution estimated using plausible values. Note that the distribution is skewed which would bias MML estimation if, as usual, a normal distribution is assumed.

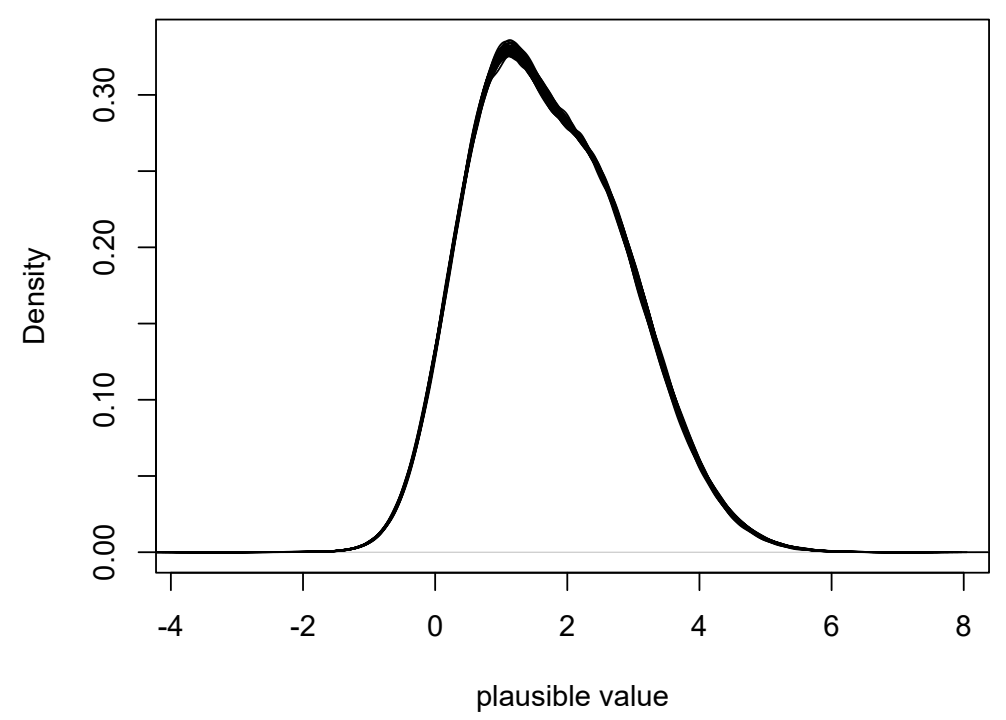

Figure 6. Densities for 50 draws of plausible values generated using 50 draws from the posterior distribution of item difficulties with burn-in 300 and step 100

\section{Discussion}

Building on the work of Maris et al. (2015), we have developed a DA Gibbs sampler for the eNRM when data are collected in incomplete designs. We have shown how the sampler can be adapted for multistage testing data and demonstrated that it is efficient enough to support a large-scale application. To this effect, we have applied the theory provided by Zwitser and Maris (2015) to Bayesian estimation, extended 
it to models for polytomous responses, and implemented it in the dexterMST R package.

Given that MST data can be analyzed with conditional or marginal ML: What are the benefits of the DA Gibbs sampler? The DA Gibbs sampler can be seen as the Bayesian equivalent of CML estimation. Both have a computational cost that is independent of the number of respondents, and are robust against misspecification of the latent variable distribution (since none needs to be specified). This furnishes an important advantage over MML estimation which is well-known to produce biased results when the ability distribution is misspecified (Zwitser \& Maris, 2015). The advantage of a, Markov chain Monte Carlo, Bayesian approach compared to ML estimation is that one does not need to compute and invert a matrix of second derivatives to obtain standard errors. As this computation is both expensive and, potentially, numerically unstable when the number of parameters is large, this is a practically significant advantage. With a Bayesian approach one simply estimates the posterior variance-covariance matrix directly from the Markov chain.

Our real data example nicely illustrated that MST can be very effective compared to linear testing. We reduced a linear test of 75 items as a MST of only 45 items in each booklet and found the latter to be almost equally reliable. MST is simpler and more cost-efficient than computerized adaptive testing (CAT) and as we've illustrated, the items can be calibrated after data collection. A limited pretest to weed out poor items and provide a rough ordering of items by difficulty will in many cases 
suffice to create an MST design. Moreover, all the design and content constraints that go with high stakes testing don't need to be dealt with whilst testing. We found the DA Gibbs sampler to be well-suited for the calibration of MST data. A design with more stages would probably be more suitable in practice but this poses no additional difficulties. Given the available space, we have limited the discussion to routing rules based on observed module scores but the theory is easily extended to more complex rules. Specifically, we can choose between routing based on the score on the last module or the score on all previous modules where the latter is equivalent to routing on estimated ability. A random element as used, for instance, in the Programme for the International Assessment of Adult Competencies (PIACC; see e.g., Chen, Yamamoto, and von Davier (2014)), can be added by simulating responses from dummy items. Further possibilities are subject for future research.

\section{Appendix}

The present paper builds on: Cressie and Holland (1983), Maris et al. (2015), and Zwitser and Maris (2015). As a courtesy to the reader, this appendix provides the gist of these paper.

\section{Marginal to Extended}

As a starting point, we take Equation 1:

$$
p_{f}(\mathbf{x} \mid \mathbf{b}, \mathbf{a})=\int_{0}^{\infty} \prod_{i} \frac{\prod_{j}\left(b_{i j} t^{a_{i j}}\right)^{x_{i j}}}{1+\sum_{j} b_{i j} t^{a_{i j}}} f(t) d t
$$


First, we re-write the model as in Cressie and Holland (1983). That is, since $\left(\prod_{i} 1+\right.$ $\left.\sum_{j} b_{i j} t^{a_{i j}}\right)^{-1}=p(\mathbf{0} \mid \mathbf{b}, \mathbf{a}, t):$

$$
\begin{aligned}
p_{f}(\mathbf{x} \mid \mathbf{b}, \mathbf{a}) & =\left(\prod_{i} \prod_{j} b_{i j}^{x_{i j}}\right) \int_{0}^{\infty} t^{x_{++}} p(\mathbf{0} \mid \mathbf{b}, \mathbf{a}, t) f(t) d t \\
& =\left(\prod_{i} \prod_{j} b_{i j}^{x_{i j}}\right) p(\mathbf{0} \mid \mathbf{b}, \mathbf{a}) \int_{0}^{\infty} t^{x_{+}} f(t \mid \mathbf{X}=\mathbf{0}, \mathbf{b}, \mathbf{a}) d t \\
& =\left(\prod_{i} \prod_{j} b_{i j}^{x_{i j}}\right) p(\mathbf{0} \mid \mathbf{b}, \mathbf{a}) \mathcal{E}\left[T^{x_{++}} \mid \mathbf{X}=\mathbf{0}, \mathbf{b}, \mathbf{a}\right] \\
& =\left(\prod_{i} \prod_{j} b_{i j}^{x_{i j}}\right) \mu \lambda_{x_{+}}
\end{aligned}
$$

with $x_{++}=\sum_{i} \sum_{j} a_{i j} x_{i j}$ the test score, $\lambda_{s}=\mathcal{E}\left[T^{s} \mid \mathbf{X}=\mathbf{0}, \mathbf{b}, \mathbf{a}\right]$, and $\mu=p(\mathbf{0} \mid \mathbf{b}, \mathbf{a})$.

Second, probabilities must sum to one and Maris et al. (2015) impose the constraint:

$$
\begin{aligned}
\mu & =\frac{1}{\sum_{x} \lambda_{x_{++}}\left(\prod_{i} \prod_{j} b_{i j}^{x_{i j}}\right)} \\
& =\frac{1}{\sum_{s} \lambda_{s}\left(\sum_{\mathbf{x} \rightarrow s} \prod_{i} \prod_{j} b_{i j}^{x_{i j}}\right)} \\
& =\frac{1}{\sum_{s} \lambda_{s} \gamma_{s}(\mathbf{b})}
\end{aligned}
$$

This gives us the manifest probabilities in Equation 2.

\section{The problem with MST: The case of the Rasch model}

Let us simplify matters and assume a Rasch model. We look at a single booklet with $m$ items and suppress the booklet index. If there are no constraints on the scores, the likelihood, for a person $p$, would be:

$$
p\left(\boldsymbol{x}_{p}\right)=\frac{\prod_{i} b_{i}^{x_{p i}} \lambda_{x_{p+}}}{\sum_{s} \gamma_{s}(\mathbf{b}) \lambda_{s}}
$$


where $b_{i}$ is the item easiness parameter, or $\exp \left(-\delta_{i}\right)$ in the usual parameterization, $x_{p i}$ indicates whether the answer of person $p$ to item $i$ was correct or not and the test score $x_{p+}$ is simply the number of correct answers given by person $p$.

What is the problem with MST? Note that the denominator is a normalizing constant. That is,

$$
\sum_{s} \gamma_{s}(\mathbf{b}) \lambda_{s}=\sum_{s}\left(\sum_{x \rightarrow s} \prod_{i} b_{i}^{x_{i}}\right) \lambda_{s}=\sum_{x}\left(\prod_{i} b_{i}^{x_{i}} \lambda_{x_{+}}\right)
$$

with $\sum_{\boldsymbol{x}}$ the sum of the numerator over all $2^{m}$ possible response patterns. The snag is that some of these response patterns are excluded under an MST design. Consider, for example, the two-stage design in Figure 2 and assume $p$ took the easy booklet. The routing rules imply that $x_{+}^{[\text {start }}<10$. Response patterns where $x_{+}^{\text {[start] }} \leq 10$ are impossible and the normalizing constant must sum over response patterns where $x_{+}^{[\mathrm{start}]}<10$.

How do we do this? We first re-write the normalizing constant to sum over the modules:

$$
\begin{aligned}
\sum_{s}\left(\sum_{x \rightarrow s} \prod_{i} b_{i}^{x_{i}}\right) \lambda_{s} & =\sum_{s} \sum_{s_{1}+s_{2}=s}\left(\sum_{x^{[\text {start }} \rightarrow s_{1}} \prod_{i \in \text { start }} b_{i}^{x_{i}} \sum_{x^{[\text {easy }]} \rightarrow s_{2}} \prod_{i \in \text { easy }} b_{i}^{x_{i}}\right) \lambda_{s} \\
& =\sum_{s} \sum_{s_{1}+s_{2}=s}\left(\gamma_{s_{1}}\left(\boldsymbol{b}^{[\text {start }]}\right) \gamma_{s_{2}}\left(\boldsymbol{b}^{[\text {[easy }]}\right)\right) \lambda_{s}
\end{aligned}
$$

where $s_{1}$ and $s_{2}$ are scores on the start and the easy module. Then, we restrict the possible values of $s_{1}$ and $s_{2}$ according to the MST design. That is, $s_{2}<10$, while $s_{1}$ is unrestricted. 


\section{References}

ACT. (2019). The ACT technical manual. Author Iowa City, IA.

Bechger, T. M., Koops, J., Partchev, I., \& Maris, G. (2019). dexterMST: CML calibration of multistage tests [Computer software manual]. Retrieved from https://CRAN.R-project.org/package=dexterMST (R package version 0.2.0)

Bechger, T. M., Maris, G., Verstralen, H., \& Béguin, A. A. (2003). Using classical test theory in combination with item response theory. Applied psychological measurement, $27(5), 319-334$.

Bejar, I. I. (2014). Past and future of multistage testing in educational reform. Computerized multistage testing: Theory and applications. New York: Chapman $\mathcal{G}$ Hall.

Bock, R. (1972). Estimating item parameters and latent ability when responses are scored in two or more nominal categories. Psychometrika, 37, 29-51.

Chalmers, R. P. (2012). mirt: A multidimensional item response theory package for the R environment. Journal of Statistical Software, 48(6), 1-29. doi: 10.18637/jss.v048.i06

Chen, H., Yamamoto, K., \& von Davier, M. (2014). Controlling multistage testing exposure rates in international large-scale assessments. Computerized multistage testing: Theory and applications, 391-409.

Cressie, N., \& Holland, P. (1983). Characterizing the manifest probabilities of latent trait models. Psychometrika, 48, 129-141.

Hendrickson, A. (2007). An NCME instructional module on multistage testing. Educational Measurement: Issues and Practice, 26(2), 44-52.

Maris, G., Bechger, T., Koops, J., \& Partchev, I. (2019). dexter: Data man- 
agement and analysis of tests [Computer software manual]. Retrieved from https://CRAN.R-project.org/package=dexter (R package version 1.0.1)

Maris, G., Bechger, T. M., \& San-Martin, E. (2015). A Gibbs Sampler for the (Extended) Marginal Rasch Model. Psychometrika, 80(4), 859-879.

Marsman, M., Maris, G., Bechger, T., \& Glas, C. (2016). What can we learn from plausible values? psychometrika, $81(2), 274-289$.

Tanner, M. A., \& Wong, W. H. (1987). The calculation of posterior distributions by data augmentation. Journal of the American statistical Association, 82(398), 528-540.

van Rijn, P. (2014). Reliability of multistage tests using item response theory. Computerized multistage testing: Theory and applications, 251-263.

Verhelst, N. D., \& Glas, C. A. (1995). The one parameter logistic model. In Rasch models (pp. 215-237). Springer.

Verhelst, N. D., Glas, C. A. W., \& van der Sluis, A. (1984). Estimation Problems in the Rasch Model: The Basic Symmetric Functions. Computational Statistics Quarterly, $1(3), 245-262$.

Yan, D., Lewis, C., \& von Davier, A. A. (2014). Overview of computerized multistage tests. Computerized multistage testing: Theory and applications. New York: Chapman $\mathcal{G}$ Hall.

Zenisky, A., Hambleton, R. K., \& Luecht, R. M. (2009). Multistage testing: Issues, designs, and research. In Elements of adaptive testing (pp. 355-372). Springer.

Zwitser, R. J., \& Maris, G. (2015). Conditional statistical inference with multistage testing designs. Psychometrika, 80(1), 65-84. 